\title{
ON THE MOMENTAL CONSTANTS OF A SUMMABLE FUNCTION*
}

\author{
BY \\ R. E. LANGER
}

I. INTRODUCTION

The definition of the momental constants $\nu_{n}$ of a summable function $f(x)$, and the first deductions concerning their relations with the generating function $f$ were given by Haskins in 1916. $\dagger$ Proofs were supplied by both Jackson $\ddagger$ and Van Vleck $\S$ for the fundamental theorem enunciated by Haskins concerning these constants. Van Vleck, moreover, by his definition and use of the "measure function" showed the existence of a relation between the momental constants of any one function and the moments of another associated with it.

The point of view maintained in the papers cited is roughly this: given a function $f(x)$ (or a pair of functions $f(x)$ and $\varphi(x)$ ), to determine certain of its (or their) characteristics by a study of the set (or sets) of momental constants. The problem dealt with in the present paper is on the other hand that of deducing a set of necessary and sufficient conditions that an enumerable set of constants be momental constants, the generating function to be defined on an arbitrarily chosen interval and to possess certain specified characteristics.

Section II in the following contains the deduction of certain necessary conditions. In Section III the sufficiency of these conditions is established. This end is attained by drawing upon Stieltjes' classic solution of the "problem of moments," and constructing theoretically through its use a function having the given constants as its momental constants. The function so derived is monotonic and as such is a typical representative of its class under Haskins' classification of summable functions. For the basis of this classification and for a statement of the significance of the momental constants the reader is referred to the original paper by Haskins.||

\section{The Deduction of CERTAIN NECESSARy CONDItions that a SET OF CONSTANTS BE MOMENTAL CONSTANTS}

1. Introduction. In this section a function $f(x)$ of specified character is considered and certain relations satisfied by its momental constants are

\footnotetext{
* Presented to the Society, February 28, 1925; received by the editors in January, 1925.

$\dagger$ These Transactions, vol. 17 (1916), p. 181.

‡ These Transactions, vol. 17 (1916), p. 178.

\& These Transactions, vol. 18 (1917), p. 326.

|| Loc. cit., pp. 185 and 194.
} 
deduced. These relations are independent of the choice of $f(x)$, subject only to the enumerated specifications. Hence they constitute conditions which must necessarily be fulfilled by any set of constants if they are to be the momental constants of a function of the kind in question.

2. Character of functions considered. Suppose given any function $f(x)$ possessed of the following characteristics :

$(A)$ it is a real, singled-valued function of the real variable $x$, defined at every point of an interval $a \leqq x \leqq b$;

$(B)$ it is integrable in the sense of Lebesgue over the interval $(a, b)$;

$(C)$ it has on the interval $(a, b)$ a finite upper measurable bound $H^{*}$ and a finite lower measurable bound $h$.

3. The momental constants. The hypotheses $(B)$ and $(C)$ imply the summability over $(a, b)$ of the functions $\{f(x)\}^{n}$ for all non-negative integral values of $n$. It is assured, therefore, that the constants

$$
\nu_{n}=\frac{1}{b-a} \int_{a}^{b}\{f(x)\}^{n} d x \quad(n=0,1,2, \cdots),
$$

exist. They are by definition the momental constants of $f(x)$ on $(a, b)$, and it is to a deduction of relations satisfied by them that we proceed.

4. A first necessary condition. Setting $n=0$ in (1) we obtain the fact that

$$
\nu_{0}=1
$$

5. A second necessary condition. From hypotheses $(A)$ and $(C)$ it follows that the function $\{f(x)\}^{2}$ is non-negative and is possessed on $(a, b)$ of a finite upper measurable bound. As such it fulfils the hypotheses of a theorem by Haskins $\dagger$ which asserts for the case in hand that if $\mu_{n}$ is the $n$th momental constant of $\{f(x)\}^{2}$ and if $L^{2}$ is the upper measurable bound of $\{f(x)\}^{2}$ then $\lim _{n \rightarrow \infty} \mu_{n} / \mu_{n-1}=L^{2}$. Inasmuch as the substitution of $\{f(x)\}^{2}$ for $f(x)$ in (1) is equivalent to the substitution of $2 n$ for $n$, it is clear that $\mu_{n}=\nu_{2 n}$. We have, therefore, as a second result

$$
\lim _{n \rightarrow \infty} \frac{\nu_{2 n}}{\nu_{2 n-2}}=L^{2}
$$

The constant $L^{2}$ is readily seen to be identical with the larger of the two numbers $H^{2}$ and $h^{2}$.

* For the definition of the measurable bounds see Haskins, loc. cit., p. 184.

† Loc. cit., p. 188. 
6. Deduction of a relation between the momental constants and Stieltjes' moments. For use in the further deductions let the function $F(x)$ be defined by the identity

$$
f(x)+L \equiv F(x)
$$

Since $L \geqq|h|$, the lower measurable bound of $F(x)$ on $(a, b)$ is positive or zero. It is with the purpose of introducing such a function to replace $f(x)$ in our considerations that the substitution (2) is made.

By virtue of relations (2) and (1) we have now

$$
\nu_{n}=\frac{1}{b-a} \int_{a}^{b}\{F(x)-L\}^{n} d x
$$

or, upon expanding the integrand,

$$
\nu_{n}=\frac{1}{b-a} \sum_{j=0}^{n}\left(\begin{array}{l}
n \\
j
\end{array}\right)(-L)^{j} \int_{a}^{b}\{F(x)\}^{n-j} d x .
$$

The Lebesgue integrals in this expression may be transformed into Stieltjes' integrals by the following method due to Van Vleck.*

The identical relation

$$
\psi(y) \equiv m E(F(x) \leqq y) \dagger
$$

defines a real single valued function of the real variable $y$ which is known as the measure function of $F(x)$. It is seen to measure the distribution of the functional values of $F(x)$ over the range $-\infty<y<\infty$. Now

$$
m E(F(x)<0)=0 \text {, and } m E(F(x)>H+L)=0 . \ddagger
$$

The structure of the Lebesgue integrals in question is, therefore, explicitly given by the formulas

$$
\int_{a}^{b}\{F(x)\}^{i} d x\left\{\begin{array}{l}
=\lim \sum_{0}^{H+L} m E\left(y_{i-1}<F(x) \leqq y_{i}\right)+m E(F(x)=0), \text { for } j=0, \\
=\lim \sum_{0}^{B+L} y_{i}^{j} m E\left(y_{i-1}<F(x) \leqq y_{i}\right), \text { for } j=1,2, \ldots . .
\end{array}\right.
$$

* Loc. cit., p. 327.

† The difference between this formula and Van Vleck's is accounted for by the fact that he considers only bounded functions while here $F(x)$ is not so restricted.

$\ddagger$ From the definition of the measurable bounds. 
Since the element under the sign of summation in terms of the function $\psi(y)$ is precisely

$$
y_{i}^{i}\left\{\psi\left(y_{i}\right)-\psi\left(y_{i-1}\right)\right\}=y_{i}^{i} \Delta_{i} \psi(y)
$$

we have the identities

(5) $\int_{a}^{b}\{F(x)\}^{i} d x\left\{\begin{array}{l}=\int_{0}^{B+L} d \psi(y)+\psi(0), \text { for } j=0, \\ =\int_{0}^{B+L} y^{i} d \psi(y), \text { for } j=1,2, \ldots .\end{array}\right.$

From (4), $\psi(y)$ is seen to be a monotonic non-decreasing function. It may be considered, therefore, to define a distribution of mass over the positive axis of $y$, the value $\psi(y)$ representing for any $y$ the mass on the interval $(0, y)$. Denoting by $c_{i}$ the $j$ th moment of this distribution about $y=0$ we have, since $\psi(y)$ is constant for $y>H+L$,

$$
c_{j}= \begin{cases}\psi(H+L), & \text { for } j=0, \\ \int_{0}^{B+L} y^{j} d \psi(y), & \text { for } j=1,2, \ldots .\end{cases}
$$

The substitution of (6) into (5) and (3) produces the relation

$$
\nu_{n}=\frac{1}{b-a} \sum_{j=0}^{n}\left(\begin{array}{c}
n \\
j
\end{array}\right)(-L)^{i} c_{n-i} .
$$

On the other hand the substitution of (2) into (5) and the expansion of the result yields in virtue of (6) the inverse relation, namely

$$
c_{n}=(b-a) \sum_{j=0}^{n}\left(\begin{array}{c}
n \\
j
\end{array}\right) L^{i} \nu_{n-i} .
$$

By means of formula (8) any relation subsisting between the constants $c_{n}$ may be translated into a relation fulfilled by the constants $\nu_{n}$. The following deduction of such a relation was given (except for small modifications) by Stieltjes.*

7. Relations satisfied by Stieltjes' moments. 'The quadratic form

$$
F_{p, m}=\int_{0}^{H+L} y^{p}\left\{T_{0}+y T_{1}+\cdots+y^{m} T_{m}\right\}^{2} d \psi(y)+\delta_{0, p} \psi(0) T_{0}^{2},
$$

-Annales de la Faculté des Sciences de Toulouse, vol. 8 (1894), p. J21, or Crutres Completes, vol. 2, p. 422. 
in which $\delta_{0, p}=0$ for $p \neq 0$ and $\delta_{0,0}=1$, is expressible alternatively in the forms

$$
\begin{aligned}
F_{p, m} & =\int_{0}^{B+L} p \sum_{i, j=0}^{m} T_{i} T_{j} y^{i+i} d \psi(y)+\delta_{0, p} \psi(0) T_{0}^{2} \\
& =\sum_{i, j=0}^{m} T_{i} T_{i} \int_{0}^{B+L} y^{p+i+j} d \psi(y)+\delta_{0, p} \psi(0) T_{0}^{2},
\end{aligned}
$$

or by virtue of $(6)$ in the form

$$
F_{p, m}=\sum_{i, j=0}^{m} c_{p+i+j} T_{i} T_{i} .
$$

From the first expression for $F_{p, m}$ above it is clear, however, that the form is positive definite. Consequently its determinant is positive, and reading this determinant from the last expression given we have

$$
D_{p, m}=\left|\begin{array}{cccccc}
c_{p} & c_{p+1} & \cdot & \cdot & \cdot & c_{p+m} \\
c_{p+1} & c_{p+2} & \cdot & \cdot & \cdot & \cdot \\
\cdot & \cdot & \cdot & \cdot & \cdot & \cdot \\
\cdot & \cdot & \cdot & \cdot & \cdot & \cdot \\
\cdot & \cdot & \cdot & \cdot & \cdot & \cdot \\
c_{p+m} & \cdot & \cdot & \cdot & \cdot & c_{p+2 m}
\end{array}\right|>0 .
$$

This result obviously holds for $p$ and $m$ any positive integers or zero. It holds then in particular for $p=0$ and $p=1$. $^{*}$

8. Further necessary conditions on the $\nu_{n}$. The substitution from (8) in (9) yields a further relation among the momental constants $\nu_{n}$. To facilitate the reduction of this relation we shall use in place of formula (8) an equivalent symbolic form obtained as follows. Comparing the sum in (8) with the polynomial $(u+L)^{n}$ it is seen that the former may be obtained from the latter by replacing each power of $u$ by the constant $\nu$ with the corresponding subscript, i. e. by replacing $u^{i}$ by $\nu_{i}, i=0,1,2, \cdots$. If it is agreed to indicate that this change is to be made in a given polynomial by enclosing in brackets, [ ], we may write (8) in the form

$$
c_{n}=(b-a)\left[(u+L)^{n}\right] .
$$

* The converse, namely, if $D_{0, m}$ and $D_{1, m}$ are positive for $m=0,1,2, \cdots$ then $D_{p, m}$ is positive for all non-negative integral values of $p$ and $m$, is not required in the reasoning of this paper. It can be shown, however, by the use of the determinant formula (8) page 26 of H. Weber's Lehrbuch der Algebra, Klein Ausgabe, Braunschweig, 1921. 
Substituting from (10) in the determinant of (9) for $p=0$ and removing the factor $(b-a)$ from each row we obtain the form

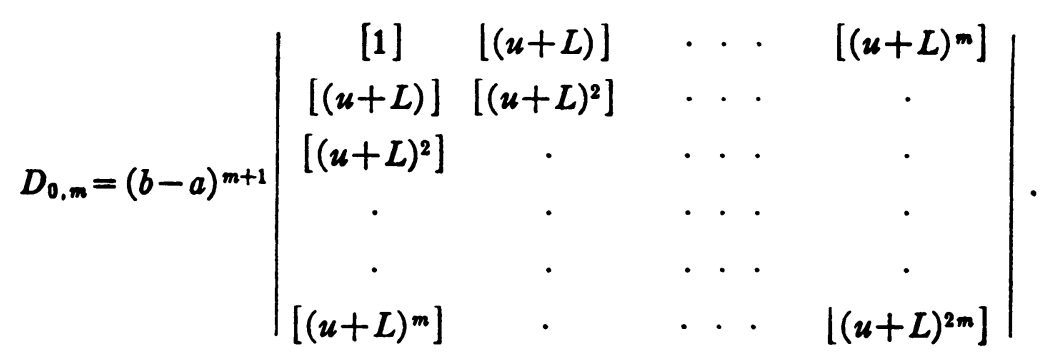

This may be simplified by replacing the $j$ th column successively for $j=m+1, m, \cdots, 2$, by the combination [column $j-$ column $(j-1)$ ]. Because of the relation

$$
\left[u^{s}(u+L)^{r}\right]-L\left[u^{s}(u+L)^{r-1}\right]=\left[u^{\sigma+1}(u+L)^{r-1}\right],
$$

which is easily verified, the net result of the operation is to replace in each element of the columns operated on one factor $(u+L)$ within brackets by a factor $u$. A repetition of the operation in obvious fashion on both columns and rows ultimately results in replacing all factors $(u+L)$ in this way. Dropping the symbolism and setting

we have the result

$$
A_{m}=D_{0, m} /(b-a)^{m+1}
$$

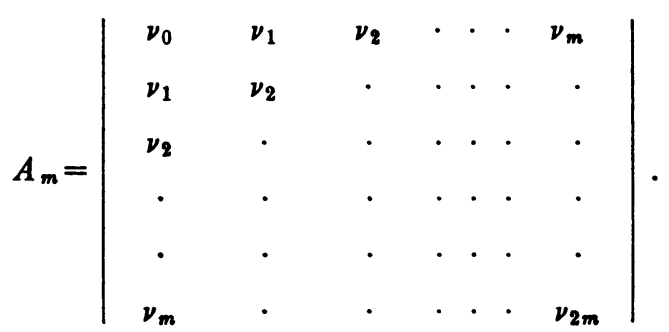

Again, substituting from (10) in the determinant of (9) for $p=1$ we obtain the form

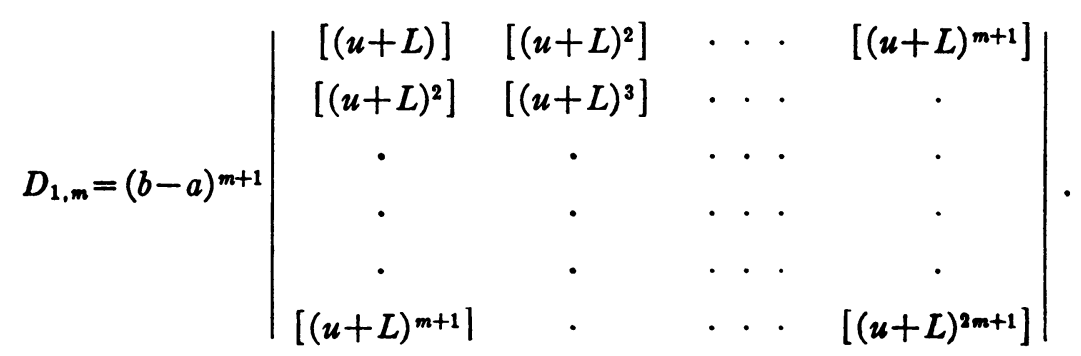


The same sequence of operations as was resorted to in the preceding case reduces this to the form

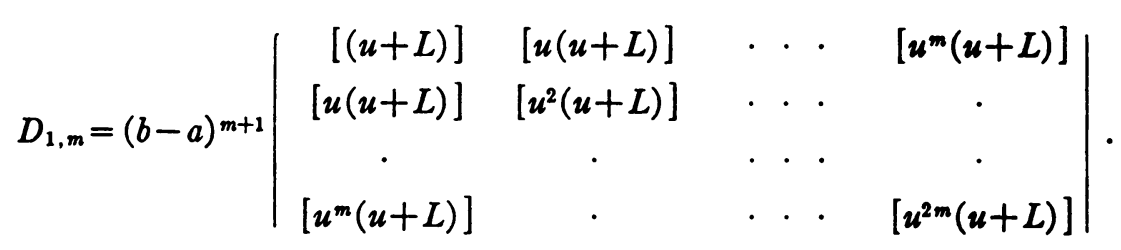

A further reduction is attained by replacing this $(m+1)$-rowed determinant by an equivalent one of $(m+2)$ rows, namely by

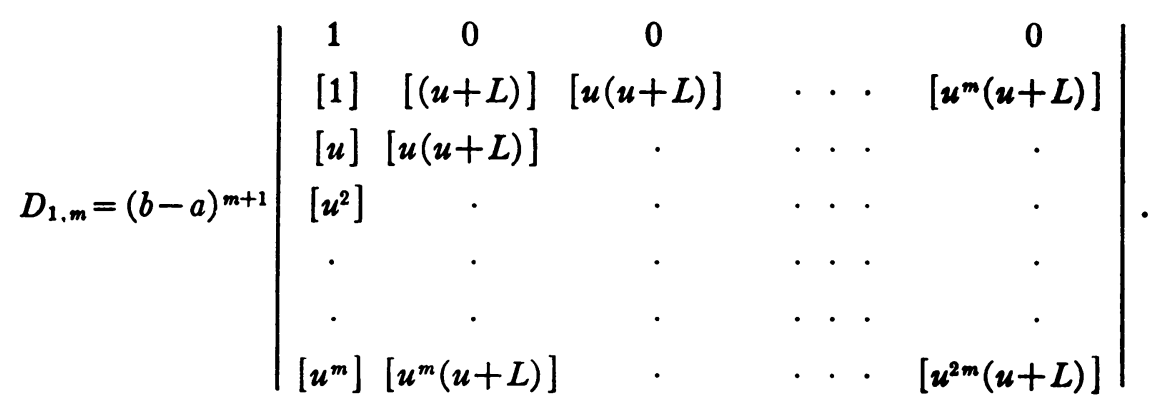

Performing now once more on the columns successively for $j=2,3, \cdots$, $(m+2)$, the same operation as was previously employed results in replacing the remaining factors $(u+L)$ by $u$. Dropping the symbolism and setting

$$
B_{m}=D_{1, m} /(b-a)^{m+1}
$$

we have

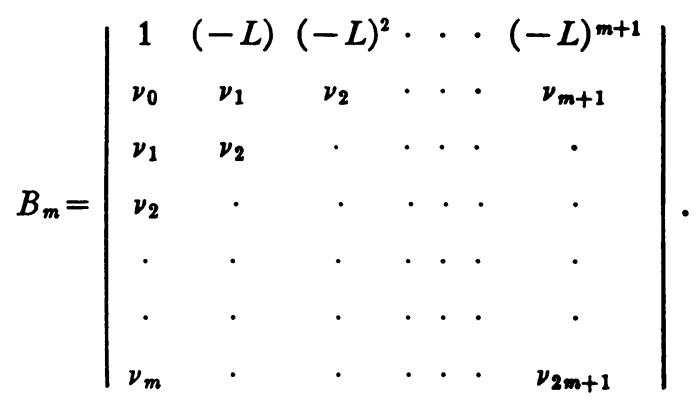

Since $A_{m}$ and $B_{m}$ differ from the positive determinants $D_{0, m}$ and $D_{1, m}$ respectively only by the positive factor $(b-a)^{m+1}$ we have as a third relation

(c)

$$
A_{m}>0, \quad B_{m}>0 \quad(m=0,1,2, \cdots)
$$


Relations $(a),(b)$, and $(c)$ together comprise a set of necessary conditions on any set of constants $\nu_{n}$ if they are to be the momental constants of a function possessed of properties $(A),(B)$, and $(C)$.

\section{Proof of the sufficiency of the set of conditions deduced}

9. Introduction. In this section an enumerably infinite set of constants $\nu_{n}$ satisfying the relations $(a),(b)$, and $(c)$ will be assumed as given, together with an interval $a \leqq x \leqq b$ for the real variable $x$. A function $f(x)$ defined on $(a, b)$ and possessing the $\nu_{n}$ as its momental constants will be constructed, and it will be shown that this function is possessed of the characteristics $(A),(B)$, and $(C)$. With the success of this procedure the sufficiency of the conditions imposed upon the constants $\nu_{n}$ is established.

10. Hypotheses concerning the constants $\nu_{n}$. Suppose given the set of constants $\nu_{n}$, satisfying the relations

$$
\begin{gathered}
\nu_{0}=1, \\
\lim _{n \rightarrow \infty} \frac{\nu_{2 n}}{\nu_{2 n-2}}=L^{2},
\end{gathered}
$$

(c) $A_{m}=\left|\begin{array}{ccccc}\nu_{0} & \nu_{1} & \cdots & \cdot & \nu_{m} \\ \nu_{1} & \nu_{2} & \cdots & . & \cdot \\ \nu_{2} & \cdot & . & . & \cdot \\ \cdot & \cdot & \cdot & \cdot & \cdot \\ \cdot & \cdot & \cdots & \cdot & \cdot \\ \nu_{m} & \cdot & \cdots & \cdot & \cdot \\ \nu_{2 m}\end{array}\right|>0$,

$$
B_{m}=\left|\begin{array}{cccc}
1 & (-L) & \cdots & (-L)^{m+1} \\
\nu_{0} & \nu_{1} & \cdots & \nu_{m+1} \\
\nu_{1} & \nu_{2} & \cdots & \cdot \\
\cdot & \cdot & \cdots & \cdot \\
\cdot & \cdot & \cdots & \cdot \\
\nu_{m} & \cdot & \cdots & \cdot \\
\nu_{2 m+1}
\end{array}\right|>0
$$

11. Introduction of a related set of constants. Consider first the conditions $(c)$. The reverse of the operations through which the forms $A_{m}$ and $B_{m}$ were deduced in the preceding section show these determinants to differ only by a positive factor respectively from the determinants $D_{0, m}$ and $D_{1, m}$ as given by formulas (11) and (12). Let the new set of constants $c_{n}(n=$ $0,1,2, \cdots)$ be defined now by means of formula (8), namely

$$
c_{n}=(b-a) \sum_{j=0}^{n}\left(\begin{array}{l}
n \\
j
\end{array}\right) L^{i \nu_{n-i}},
$$

or its equivalent (10),

$$
c_{n}=(b-a)\left[(u+L)^{n}\right] .
$$


The steps following the derivation of the relation (9) in the preceding chapter are now reversible. Retracing them, the conditions $(c)$ on the set of constants $\nu_{n}$ are found to be equivalent to the conditions

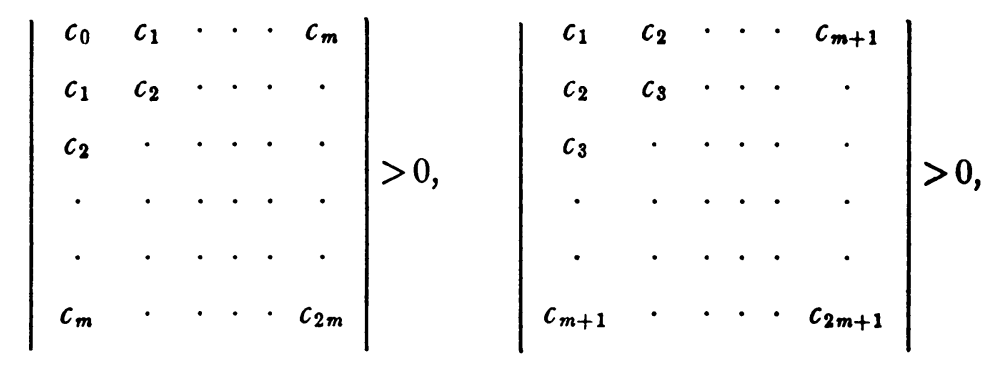

on the constants of the associated set $c_{n}$.

12. Outline of Stieltjes' solution of the problem of moments. The conditions (13) on a set of constants are familiar ones. Stieltjes showed* that when they are fulfilled then the series

$$
\frac{c_{n}}{z}-\frac{c_{1}}{z^{2}}+\frac{c_{2}}{z^{3}}-\cdots
$$

is the formal expansion of a continued fraction of the type

$$
\frac{1}{a_{1} z}+\frac{1}{a_{2}}+\frac{1}{a_{3} z}+\cdots,
$$

with positive coefficients $a_{i}$. On the other hand he showed how the convergents of such a fraction may be utilized in the construction o a sequence of functions $\varphi_{n}(y)$ from which an associated function $\Phi(y)$ may be determined. The existence of this function solves the "problem of moments," for it follows from its mode of derivation first that $\Phi(y)$ defines a distribution of mass in the manner of $\psi(y)$ in the preceding section and second that

$$
\int_{0}^{\infty} y^{n} d \Phi(y)=c_{n} \quad(n=0,1,2, \ldots) .
$$

Two cases are distinguished by Stieltjes in his deductions. Under the conditions (13) the ratio $c_{n+1} / c_{n}$ increases with $n . \dagger \mathrm{He}$ showed that when it approaches a finite limit the function $\Phi(y)$ is determined uniquely save for its values at its points of discontinuity. In the alternative case there are infinitely many such functions.

- Loc. cit., A n n a l e s, p. J1, CEuvres p. 402.

$\dagger$ This can be shown as follows: By the footnote on page 172, conditions (13) imply that $D_{n, 0}>0$, $D_{n+1,0}>0$, and $D_{n, 1}>0$ for all positive $n$. Hence $D_{n, 1} /\left(D_{n, 0} D_{n+1,0}\right)>0$, that is, $c_{n+2} / c_{n+1}>c_{n+1} / c_{n}$. 
We shall not distinguish at this time between the two cases, but shall summarize those of Stieltjes' results which are of importance for the purpose before us as follows. Given any set of constants $c_{n}$ satisfying the conditions (13), there exists at least one function $\Phi(y)$ which

(i) is defined and single valued for $0 \leqq y<\infty$,

(ii) is monotonic non-decreasing,

(iii) vanishes at zero, i. e. $\Phi(0)=0$,

(iv) satisfies the relations

$$
\int_{0}^{\infty} y^{n} d \Phi(y)=c_{n} \quad(n=0,1,2, \cdots) .
$$

13. The function $\psi(y)$. The properties enumerated restrict the discontinuities of $\Phi(y)$ to occur in the points of an enumerable set.* Because of this the values taken on by $\Phi(y)$ at its points of discontinuity (excepting the point $y=0$ if it is among them) may be changed at pleasure without affecting the integrals in (iv). In particular the value at every such point may be made equal to the right hand limiting value, i. e.

$$
\Phi(y)=\lim _{\substack{\epsilon \rightarrow 0 \\ \epsilon>0}} \Phi(y+\epsilon) .
$$

We shall suppose this to have been done, and since this alteration is to be made at $y=0$ also, if $\Phi(y)$ is discontinuous there we shall denote the new function so obtained by $\psi(y)$. Obviously $\psi(y)$ is possessed of properties (i) and (ii). In place of (iii) and (iv) it has the characteristics that

(iii) $^{\prime}$ it is non-negative, i. e. $\psi(0) \geqq 0$,

(iv) ${ }^{\prime}$ it satisfies the relations

$$
\begin{aligned}
& \int_{0}^{\infty} d \psi(y)+\psi(0)=c_{0}, \\
& \int_{0}^{\infty} y^{n} d \psi(y)=c_{n} \quad(n=1,2, \cdots) .
\end{aligned}
$$

In addition we have by construction that

(v) it is continuous toward the right.

14. A lemma. The end toward which our deductions point is the construction of a suitable function of $x$. The following lemma is to serve as a basis upon which such a function may be derived from the $\psi(y)$ at hand.

\footnotetext{
- See Stieltjes, loc. cit., chap. vi.
} 
LEMмA. Given a function $\omega(y)$ which is

(a) defined and single-valued for $0 \leqq y<\infty$ or $0 \leqq y \leqq Y$,

( $\beta$ ) monotonic non-decreasing,

$(\gamma)$ non-negative on its interval of definition,

( $\delta$ ) continuous to the right, $i$. e.

$$
\omega(y)=\lim _{\substack{\epsilon \rightarrow 0 \\ \epsilon>0}} \omega(y+\epsilon)
$$

Then if $M(x)$ is the measure function of $\omega(y)$, conversely $\omega(y)$ is the measure function of $M(x)$.

The function $\omega(y)$ has 0 as a lower bound, whence, by the definition of the measure function for the case in hand,

$$
M(x) \equiv m E_{y}(0 \leqq \omega(y) \leqq x) .
$$

The subscript ( $y$ in this case) is appended to the $E$ to assist one in following the reasoning. It denotes the axis along which the point set in question is measured. It is clear from the identity (14) that every measure function is possessed of the properties $(a),(\beta),(\gamma)$, and $(\delta)$ as enumerated above. If we denote the measure function of $M(x)$ by $\Omega(y)$ we have accordingly

$$
\Omega(y) \equiv m E_{x}(0 \leqq M(x) \leqq y) .
$$

Moreover, $\Omega(y)$ will have the properties $(a),(\beta),(\gamma)$, and $(\delta)$ in common with $\omega(y)$. We wish to show that $\Omega(y) \equiv \omega(y)$. Because of the right-handed continuity of both functions it will suffice to show that their values are the same at every point at which $\omega(y)$ is continuous. We shall consider separately the cases when $y$ is a value at which $\omega(y)$ is increasing and when $y$ is a value at which $\omega(y)$ is stationary.

Case 1. Let $y=y_{1}$ be a point at which (i) $\omega(y)$ is continuous, and (ii) $\omega(y)$ is increasing, i. e.,

$$
\omega\left(y_{1}\right)<\omega\left(y_{1}+\epsilon\right) \text { for every } \epsilon>0 \text {. }
$$

By hypothesis $\omega(y)$ is defined at every point of the interval $0 \leqq y \leqq y_{1}$. Moreover it is monotonic and non-negative. It follows that

$$
m E_{y}\left(0 \leqq \omega(y) \leqq \omega\left(y_{1}\right)\right)=y_{1} .
$$

Defining $x_{1}$ by the relation $x_{1}=\omega\left(y_{1}\right)$ and comparing (16) with (14) we see that

$$
\text { if } x_{1}=\omega\left(y_{1}\right) \text {, then } M\left(x_{1}\right)=y_{1} \text {. }
$$

Now since $\omega(y)$ is continuous and increasing at the point $y=y_{1}$, a small increase in $y_{1}$ is correlated with a small increase in $\omega\left(y_{1}\right)$, namely in $x_{1}$. 
Because of the relation $M\left(x_{1}\right)=y_{1}$ from (17) this means that $M(x)$ is continuous and increasing at $x=x_{1}$.

Proceeding, one sees from (14) that $M(x)$ is defined at every point of the interval $0 \leqq x \leqq x_{1}$. Inasmuch as it is also non-decreasing and non-negative the reasoning which leads from (14) to (17) may be applied again and leads from (15) to the fact

$$
\text { if } y_{1}=M\left(x_{1}\right) \text { then } \Omega\left(y_{1}\right)=x_{1} .
$$

In consequence of (17) and (18) we have

$$
\Omega\left(y_{1}\right)=\omega\left(y_{1}\right) .
$$

Case 2. Let $y$ be a point in which $\omega(y)$ is continuous but stationary, i. e.

$$
\omega(y) \equiv \omega(y+\epsilon) \text { for } 0 \leqq \epsilon<\delta .
$$

In this case $\omega(y)$ is constant throughout an interval, namely

$$
\omega(y) \equiv x_{1} \quad \text { for } \quad y_{1} \leqq y<y_{2} .
$$

From formula (14), then,

while

$$
M\left(x_{1}-0\right)=m E_{y}\left(0 \leqq \omega(y)<x_{1}\right)=y_{1},
$$

$$
M\left(x_{1}\right)=m E_{y}\left(0 \leqq \omega(y) \leqq x_{1}\right)=y_{2} .
$$

The function $M(x)$ is seen to be discontinuous in $x=x_{1}$. It takes on no values lying between $y_{1}$ and $y_{2}$ and hence

$$
m E_{x}(0 \leqq M(x) \leqq y) \equiv m E_{x}\left(0 \leqq M(x) \leqq y_{1}\right),
$$

when $y_{1} \leqq y<y_{2}$. But since $M(x)$ is defined for every $x$ on the interval $0 \leqq x \leqq x_{1}$, and is non-decreasing and non-negative, the right hand member is simply $x_{1}$. The left hand member on the other hand is $\Omega(y)$ by (15). Hence (19) yields $\Omega(y) \equiv x_{1}$ for $y_{1} \leqq y<y_{2}$. This together with the hypothesis of Case 2 gives

$$
\Omega(y) \equiv \omega(y), \text { for } y_{1} \leqq y<y_{2} .
$$

This concludes the proof of the lemma.

15. Application of the lemma. Returning now to $\psi(y)$, let its measure function be constructed and denoted by $F(x)$. This function is defined on the interval $0 \leqq x \leqq \psi(\infty)$. But the properties (i), (ii), (iii)' and (v) of $\psi(y)$ are precisely the properties $(a)$ to $(\delta)$ of the hypothesis for $\omega(y)$ in the lemma. It follows then by the lemma that $\psi(y)$ is in turn the measure function of 
$F(x)$. By the reasoning employed to establish the relation (5) we may deduce then that

$$
\int_{0}^{\psi(\infty)}\{F(x)\}^{n} d x\left\{\begin{array}{l}
=\int_{0}^{\infty} d \psi(y)+\psi(0), \text { for } n=0, \\
=\int_{0}^{\infty} \psi^{n} d \psi(y), \text { for } n=1,2, \ldots .
\end{array}\right.
$$

But because of the property (iv)' of $\psi(y)$ the right hand expressions for any $n$ are precisely $c_{n}$. Since (iv) ${ }^{\prime}$ yields also $\psi(\infty)=c_{0}$, it follows that the function $F(x)$ constructed in the manner shown satisfies the relations

$$
\int_{0}^{c_{0}}\{F(x)\}^{n} d x=c_{n} \quad(n=0,1,2, \cdots) .
$$

16. Construction of the function $f(x)$ having the $\nu_{n}$ as its momental constants. Formula (8) defines the constants $c_{n}$ in terms of the constants $\nu_{n}$. The inverse relation is given by (7), and substituting in this the value of $c_{n}$ as given by (20) we obtain

$$
\nu_{n}=\frac{1}{b-a} \sum_{j=0}^{n}\left(\begin{array}{c}
n \\
j
\end{array}\right)(-L)^{j} \int_{0}^{c_{j}}\{F(x)\}^{n-i} d x
$$

that is,

$$
\nu_{n}=\frac{1}{b-a} \int_{0}^{c_{0}}\{F(x)-L\}^{n} d x .
$$

From (8) and hypothesis $(a)$ we have, moreover, $c_{0}=(b-a)$, which substituted in (21) shows the interval of integration to be from 0 to $(b-a)$. This is changed to the given interval $(a, b)$ by further setting

$$
\begin{aligned}
x^{\prime} & =x+a, \\
F(x)-L & =f\left(x^{\prime}\right) .
\end{aligned}
$$

Upon dropping the primes on the variable of integration the resulting expression becomes

$$
\nu_{n}=\frac{1}{b-a} \int_{a}^{b}\{f(x)\}^{n} d x .
$$

The function $f(x)$ therefore has the given constants $\nu_{n}$ as its momental constants on $(a, b)$. The characteristics of $f(x)$ remain to be determined.

17. The properties of $f(x)$. A review of the mode of derivation given readily shows that $f(x)$ is possessed of the characteristics $(A)$ and $(B)$ 
enunciated at the beginning of this section. Further, we have by hypothesis $(b)$ that

$$
\lim _{n \rightarrow \infty} \frac{\nu_{2 n}}{\nu_{2 n-2}}=L^{2}
$$

But $\nu_{2 n}$ is the $n$th momental constant of the non-negative function $\{f(x)\}^{2}$. It follows by Haskins' theorem* that $\{f(x)\}^{2}$ has the upper measurable bound $L^{2}$, and hence that the measurable bounds of $f(x)$ are restricted from exceeding in absolute value the constant $L$. The function $f(x)$ is, therefore, also possessed of the property $(C)$.

Summarizing our results we have the

THEOREM. The conditions $(a),(b)$, and $(c)$ enunciated above are both necessary and sufficient that the enumerable set of constants $\nu_{n}$ be the momental constants of a function possessed of the properties $(A),(B)$, and $(C)$.

\section{Concluding Remarks}

18. Introduction. With the result of the preceding section the principal problem of this paper is solved. It is clear, however, that the procedure of Section III besides constituting a proof of the sufficiency of the conditions on the $\nu_{n}$, embodies also a theoretical method for the construction of a non-decreasing function which is continuous on the right and has the $\nu_{n}$ as its momental constants. The uniqueness of this function may be shown as follows.

19. The uniqueness of the solution. The measurable bounds of $f(x)$ on $(a, b)$ do not exceed in absolute value the constant $L$. Hence the measurable bounds of the function $F(x)$ in (18) are restricted to lie on the range from 0 to $2 L$. This implies, however, that $\psi(y)$, the measure function of $F(x)$, is constant for $y \geqq 2 L$, or in other words that the distribution of matter defined by it is confined to a finite interval. But it was shown by Van Vleck $\dagger$ that under these conditions the ratio $c_{n+1} / c_{n}$ approaches a finite limit. This is the distinguishing characteristic of Stieltjes' determinate case, namely the case in which $\Phi(y)$ is unique save for its values in the points of discontinuity. The function $\psi(y)$ satisfying the conditions enumerated in Section III is, therefore, unique.

Suppose now that $f_{2}(x)$ is distinct from $f(x)$ but shares with it the properties that its momental constants are $\nu_{n}$ and that it is non-decreasing and continuous toward the right. Because of the last named property the points in which $f(x)$ and $f_{2}(x)$ are distinct comprise a set of positive measure.

* See note on page 169.

$\dagger$ Loc. cit., p. 330. 
Hence the related functions $F(x)$ and $F_{2}(x)$ will also differ on the points of a set of positive measure and it follows that their measure functions $\psi(y)$ and $\psi_{2}(y)$ are not identical. Since they share the properties enumerated for $\psi(y)$ in Section III this is in contradiction with the uniqueness of $\psi(y)$ already shown. The hypothesis that $f_{2}(x)$ and $f(x)$ are distinct is, therefore, untenable.

If the requirement of right handed continuity is dropped the uniqueness of $f(x)$ at its points of discontinuity is lost, for an alteration of the values of $f(x)$ at these points need not impair its non-decreasing character and will not affect its Lebesgue integral. Otherwise, however, $f(x)$ remains determined and hence the deductions made constitute a new proof of the fact observed by Haskins and first proved by Jackson, ${ }^{*}$ namely that the nondecreasing function with given momental constants is determined uniquely save at the points of an enumerable set.

20. A means for obtaining the typical representative of a class of summable functions. One further observation may be made, namely, if $\bar{f}(x)$ is a bounded summable function and belongs to a certain class $C$ under Haskins' classification, $\dagger$ then $f(x)$, the measure function of the measure function of $\bar{f}(x)$, also belongs to $C$ and is a typical representative of that class.

The proof of this is immediate. If $H$ and $h$ are the bounds of $\bar{f}(x)$, and $M(y)$ is its measure function on $(a, b)$, then

$$
\int_{a}^{b}\{\bar{f}(x)\}^{n} d x=\int_{h}^{B} y^{n} d M(y)+h^{n} M(h) .
$$

By definition $f(x)$ is the measure function of $M(y)$, and since $M(y)$ satisfies the hypotheses of the lemma in Section III (a fact which is easily verified) it follows that $M(y)$ is in turn the measure function of $f(x)$. We have, therefore, also

$$
\int_{a}^{b}\{f(x)\}^{n} d x=\int_{h}^{B} y^{n} d M(y)+h^{n} M(h),
$$

that is,

$$
\int_{a}^{b}\{f(x)\}^{n} d x \equiv \int_{a}^{b}\{\bar{f}(x)\}^{n} d x,
$$

i. e. $f(x)$ has the same momental constants as $\bar{f}(x)$, and so is a member of the class $C$. Being a measure function, however, $f(x)$ is non-decreasing. This completes the proof.

* These Transactions, vol. 17 (1916), p. 179.

† See last foot note on page 168.

Dartmouth College, HANOVER, N. H. 